the highest overall persistence rate (median persistence rate $>50.3 \%$ where 47.4 months is the maximum follow up time recorded), followed by IFX and ADA (p <0.001) (figure 2).

Biological agent persistence correlated with immunomodulator co-therapy in $\mathrm{CD}\left(\mathrm{R}^{2}: 0.65 \mathrm{p}<0.001\right)$ and $\mathrm{UC}\left(\mathrm{R}^{2}: 0.50\right.$ $\mathrm{p}<0.001)$. Thiopurine co-therapy increased persistence for IFX and ADA in CD and UC $(\mathrm{p}<0.001)$ with a trend for higher persistence for UST in CD $(\mathrm{p}=0.06)$ (figures 3,4). Methotrexate decreased persistence for VDZ in UC and had no effects on IFX and ADA in UC and CD.

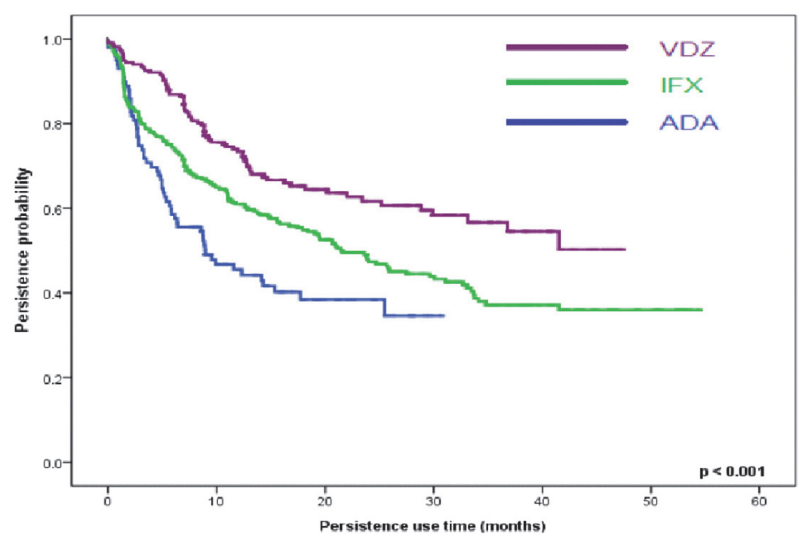

Abstract IDDF2020-ABS-0033 Figure 2 Persistence Kaplan meier graphs- Moderate-severe ulcerative colitis persistence

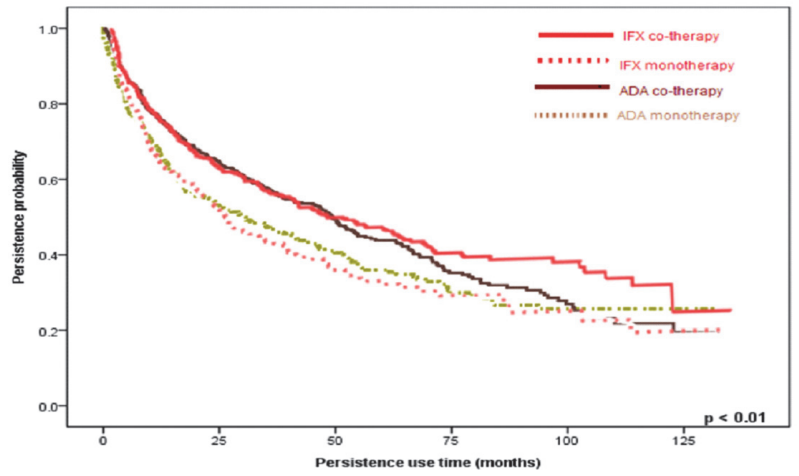

Abstract IDDF2020-ABS-0033 Figures 3 Persistence Kaplan meier graphs-Thiopurine co-therapy in Crohn's disease

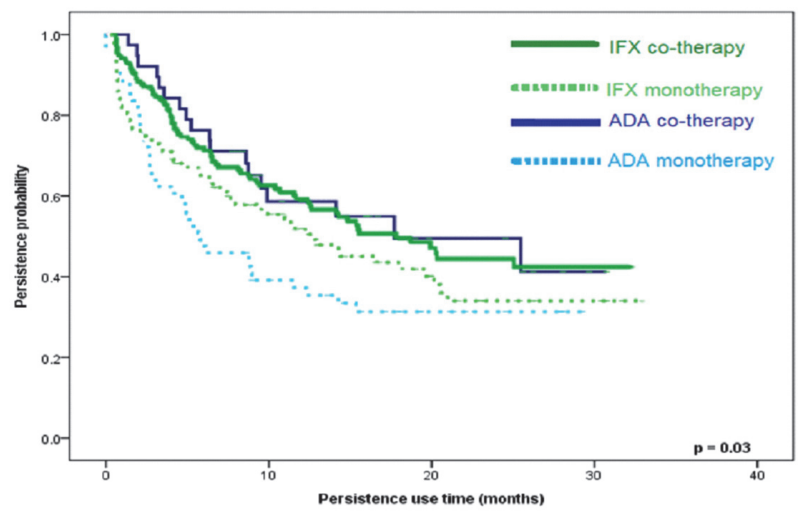

Abstract IDDF2020-ABS-0033 Figures 4 Persistence Kaplan meier graphs-Thiopurine co-therapy in Ulcerative colitis
On multivariate analysis, higher persistence was seen in males (HR: 0.75 95\%CI: 0.59-0.94) and with immunomodulator co-therapy (HR 0.77 95\% CI: 0.61-0.97, $\mathrm{p}=0.03$ ).

Conclusions This real-world data reflecting treatment effectiveness, tolerability and patient and prescriber preferences supports the use of ustekinumab and vedolizumab over antiTNF agents in CD and UC respectively to achieve high treatment persistence, possibly secondary to low immunogenicity. It also highlights the substantial increase in biological therapy persistence with thiopurine co-therapy, rather than methotrexate.

\section{IDDF2020-ABS-0165 ACCURACY AND APPLICABILITY OF THE ARTIFICIAL INTELLIGENCE INTEGRATED SOFTWARE IN Z-LINE SEGMENTATION}

${ }^{1} \mathrm{Hang}$ Dao*, ${ }^{2}$ Hung Le, ${ }^{1}$ Binh Nguyen, ${ }^{1} \mathrm{Hung}$ Nguyen, ${ }^{3} \mathrm{Huy}$ Manh, ${ }^{3} \mathrm{Kien} \mathrm{Ha},{ }^{3} \mathrm{Hai} \mathrm{Vu}$. ${ }^{1}$ Institute of Gastroenterology and Hepatology, Vietnam; ${ }^{2}$ Hanoi Medical University hospital, Vietnam; ${ }^{3}$ Hanoi University of Science and Technology, Vietnam

\subsection{6/gutjnl-2020-IDDF.7}

Background Artificial intelligence (AI) and its applications in developing software for assisting endoscopic education are novel research directions around the world in general and in Vietnam particularly. This study aims to assess the software accuracy in assisting Z-line segmentation by comparing with medical doctors' detection results and record doctors' satisfaction in scale, time-of-implementation in interactive mode, and integrated mode.

Methods This research was conducted from April 2019 to July 2020. For the development of the Z-line detection algorithm, a dataset of 533 high-definition endoscopic WLI (white-light) images in diverse forms of Z-line were collected. Preliminary assessment of the AI algorithm yielded a good IoU (Interception over Union) of $72 \%$. The software was subsequently developed in 4 modes, including manual mode, interactive mode (using Superpixels-BPT), automatic mode (using AI algorithm), and integrated mode (the combination of BPT and U-Net). 30 endoscopic images were assigned to 2 groups of doctors (naive and experienced group) for the Z-line detection using the software in 4 modes. Assessment indicators including time-of-implementation, number of mouse clicks, satisfaction in scales, and IoU metric with expert's ground-truth were taken into account.

Results The IoU metric of interactive and integrated modes in the experimental dataset deviated from $87.3 \%$ to $88.5 \%$ with no statistical difference to the IoU value of manual mode, and mean IoU metrics from the results of 2 doctor groups were over $85 \%$. The mean values of time-of-implementation in interactive mode and integrated mode were not statistically different from manual mode. The median number of mouse clicks each use in the interactive mode and the integrated mode were 24.5 and 15.5 times, respectively. The software received good feedbacks from the doctors, with the mean values of satisfaction scores of automatic mode, interactive mode and integrated mode were 7.19, 7.26, and 7.18, respectively.

Conclusions The development of the software for detecting endoscopic anatomy landmarks is a novel and feasible research direction in Vietnam. Further studies could focus on detecting some specific lesions. 